\title{
First order flow equations for nonextremal black holes in AdS (super)gravity
}

\author{
Dietmar Klemm and Marco Rabbiosi \\ Dipartimento di Fisica, Università di Milano, \\ Via Celoria 16, 20133 Milano, Italy \\ INFN, Sezione di Milano, \\ Via Celoria 16, 20133 Milano, Italy \\ E-mail: dietmar.klemm@mi.infn.it, marco.rabbiosi@mi.infn.it
}

ABSTRACT: We consider electrically charged static nonextremal black holes in $d$-dimensional Einstein-Maxwell-(A)dS gravity, whose horizon is a generic Einstein space in $d-2$ dimensions. It is shown that for this system the Hamilton-Jacobi equation is exactly solvable and admits two branches of solutions. One of them exhibits a non-simply connected domain of integration constants and does not reduce to the well-known solution for the $d=4$ BPS case. The principal functions generate two first order flows that are analytically different, but support the same general solution. One of the two sets of flow equations corresponds to those found by Lü, Pope and Vázquez-Poritz in hep-th/0307001 and (for $d=4$ and $\Lambda=0$ ) by Miller, Schalm and Weinberg in hep-th/0612308. This clarifies also the reason for the very existence of first order equations for nonextremal black holes, namely, they are just the expressions for the conjugate momenta in terms of derivatives of the principal function in a Hamilton-Jacobi formalism. In the last part of our paper we analyze how much of these integrability properties generalizes to matter-coupled $N=2$, $d=4$ gauged supergravity.

KeYwords: Black Holes, Classical Theories of Gravity, Supergravity Models

ARXIV EPRINT: 1706.05862 


\section{Contents}

1 Introduction 1

2 Static black holes in Einstein-Maxwell-(A)dS gravity 2

2.1 Electrically charged black holes 3

2.2 Effective action 3

3 Integration of the Hamilton-Jacobi equation 4

3.1 First solution 5

$\begin{array}{lll}3.2 & \text { Second solution } & 6\end{array}$

4 Matter-coupled $N=2, d=4$ gauged supergravity $\quad 7$

4.1 Prepotential $F=-i X^{0} X^{1} \quad 9$

5 Final remarks $\quad 11$

\section{Introduction}

Exact solutions to Einstein's field equations and their supergravity generalizations have been playing, and continue to play, a crucial role in many important developments in general relativity, black hole physics, integrable systems, string theory and quantum gravity. Being highly nonlinear, coupled partial differential equations, these are notoriously difficult to solve, sometimes even in presence of a high degree of symmetry, for instance in supergravity where one has typically many other fields in addition to the metric. If one imposes that some fraction of supersymmetry be preserved, the construction of solutions simplifies considerably, since one has to solve only the first order Killing spinor equations instead of the full higher order equations of motion.

In the last years however it was shown (see [1-17] for an (incomplete) list of references) that sometimes also non-BPS- and even nonextremal black holes satisfy certain first order equations, which typically arise by writing the potential of a one-dimensional mechanical system (to which the supergravity action boils down if one has enough symmetry) in terms of a 'superpotential'. ${ }^{1}$ The deeper reason behind this has remained rather obscure, since these obviously have nothing to do with supersymmetry.

Here we will elaborate on results obtained in [21], shewing for the example of EinsteinMaxwell-(A)dS gravity in arbitrary dimension that the first order flow equations satisfied by electrically charged static nonextremal black holes (found in part for instance in [1, 2])

\footnotetext{
${ }^{1}$ An analysis of nonextremal solutions was used in [18] to study Einstein-dilaton black holes. In the most general case with a vector and scalar this was done in [19], where the motivation was the computation of the finite temperature/finite density effective potential in holography. Recently the zero temperature case was analyzed in full generality in Einstein-dilaton gravity [20] in order to find the most general RG flows.
} 
are just the expressions for the conjugate momenta in terms of derivatives of the principal function $W$ in a Hamilton-Jacobi formalism [22]. Moreover, we will see that the expression for the potential in terms of a 'superpotential' is nothing else than the Hamilton-Jacobi equation for zero energy. The fact that a nonextremal black hole solution arises from a first order system via a superpotential construction is thus not surprising at all.

We will also find that (for the theory under consideration) there exist actually two different branches of solutions to the HJ equation. This leads to two distinct sets of flow equations, that share the same black hole solutions.

Guided by the structure of $W$ in the Einstein-Maxwell- $\Lambda$ case, one can try to generalize our analysis for instance to $N=2$ gauged supergravity in four dimensions, where the superpotential in the BPS case is known both for U(1) Fayet-Iliopoulos gauging [23] and for coupling to hypermultiplets, when abelian isometries of the quaternionic hyperscalar target manifold are gauged [17]. Unfortunately it turns out that the principal function $W$ for nonextremal black holes is not straightforwardly generalizable to the matter-coupled case. Nevertheless, we show (for the example of a particular prepotential) that there exist several conserved charges that allow a partial separation of variables in the HJ equation. Among these conserved charges there is the one originally introduced for ungauged supergravity in [24] and subsequently adapted to the gauged theory in [17]. Moreover, it was recently found [25] that $N=2, d=4 \mathrm{U}(1)$ Fayet-Iliopoulos gauged supergravity enjoys residual symmetries that essentially involve the stabilization of the symplectic vector of gauge couplings (FI parameters) under the action of the U-duality symmetry of the ungauged theory. This provides additional conserved charges.

The remainder of this paper is organized as follows: in the next section, we consider Einstein-Maxwell-(A)dS gravity in arbitrary dimension, adopt an ansatz for electrically charged static black holes whose event horizon is a generic $(d-2)$-dimensional Einstein space, and determine the one-dimensional effective action from which one can derive the equations of motion. In section 3 we integrate the Hamilton-Jacobi equation associated to this mechanical system in full generality and show that there are two branches of solutions. This leads to two different sets of first order flow equations, one of which coincides with that found in [1]. In section 4 we analyze how much of these integrability properties ${ }^{2}$ can be generalized to matter-coupled $N=2, d=4$ gauged supergravity. We conclude in 5 with some final remarks.

\section{Static black holes in Einstein-Maxwell-(A)dS gravity}

We consider $d$-dimensional Einstein-Maxwell-(A)dS gravity, whose action is given by

$$
S=\frac{1}{16 \pi G_{d}} \int \mathrm{d}^{d} x \sqrt{-g}\left(R-F_{\mu \nu} F^{\mu \nu}-2 \Lambda\right),
$$

with $d>3$. This is the simplest model that can be embedded (at least for some $d$ ) in $N=2$ gauged supergravity. The equations of motion following from (2.1) are

$$
R_{\mu \nu}-\frac{1}{2} R g_{\mu \nu}+\Lambda g_{\mu \nu}=2\left(F_{\mu \sigma} F_{\nu}{ }^{\sigma}-\frac{1}{4} g_{\mu \nu} F_{\sigma \rho} F^{\sigma \rho}\right), \quad \nabla_{\mu} F^{\mu \nu}=0,
$$

\footnotetext{
${ }^{2}$ Integrability in presence of a cosmological constant was studied before in [26-29].
} 
where $F=\mathrm{d} A$. For future convenience we report the trace and the traceless part of the Einstein equations that respectively read

$$
\begin{array}{r}
R-\frac{2 d}{d-2} \Lambda-\frac{d-4}{d-2} F^{\mu \nu} F_{\mu \nu}=0, \\
R_{\mu \nu}-2 F_{\mu}{ }^{\sigma} F_{\nu \sigma}-\frac{2}{d-2} \Lambda g_{\mu \nu}+\frac{1}{d-2} g_{\mu \nu} F^{\sigma \rho} F_{\sigma \rho}=0 .
\end{array}
$$

\subsection{Electrically charged black holes}

In what follows we shall consider electrically charged static black holes whose horizon is a $(d-2)$-dimensional Einstein space. ${ }^{3}$ The metric and the gauge field have the form

$$
\mathrm{d} s_{d}^{2}=-e^{-2(d-3) U} \mathrm{~d} t^{2}+e^{2 U-2(d-4) \psi} \mathrm{d} r^{2}+e^{2(U+\psi)} \mathrm{d} \Omega_{\kappa, d-2}^{2}, \quad A=A_{t} \mathrm{~d} t,
$$

where the functions $U, \psi$ and $A_{t}$ depend only on the coordinate $r$. The metric in (2.4) has the warped product structure

$$
\mathrm{d} s_{d}^{2}=\tilde{g}_{a b} \mathrm{~d} x^{a} \mathrm{~d} x^{b}+f^{2}(x) \hat{g}_{i j} \mathrm{~d} y^{i} \mathrm{~d} y^{j},
$$

where the $(d-2)$-dimensional fiber with metric $\hat{g}_{i j} \mathrm{~d} y^{i} \mathrm{~d} y^{j}=\mathrm{d} \Omega_{\kappa, d-2}^{2}$ is a generic Einstein space, i.e., $\hat{R}_{i j}=(d-3) \kappa \hat{g}_{i j}$. The nonvanishing components of the Ricci tensor in $d$ dimensions are thus given by [30]

$$
\begin{aligned}
& R_{a b}=\tilde{R}_{a b}-\frac{d_{F}}{f} \tilde{\nabla}_{a} \tilde{\nabla}_{b} f \\
& R_{i j}=\hat{R}_{i j}-\hat{g}_{i j}\left(f \tilde{\nabla}_{a} \tilde{\nabla}^{a} f+\left(d_{F}-1\right) \tilde{g}^{a b} \partial_{a} f \partial_{b} f\right),
\end{aligned}
$$

where $d_{F}>1$ is the dimension of the fiber and $\tilde{\nabla}_{a}$ denotes the covariant derivative constructed with the Levi-Civita connection for $\tilde{g}_{a b}$.

\subsection{Effective action}

The Maxwell equations for the ansatz (2.4) are solved by

$$
F=-Q e^{-2(d-3)(U+\psi)} \mathrm{d} t \wedge \mathrm{d} r
$$

where $Q$ is an integration constant corresponding to the electric charge. Using (2.6) it is straightforward to shew that the Einstein equations (2.3) boil down to three ordinary differential equations that can be derived form the one-dimensional effective action

$$
S_{\text {eff }}=\int \mathrm{d} r L=\int \mathrm{d} r\left(e^{2(d-3) \psi}\left(U^{\prime 2}-\psi^{\prime 2}\right)-V_{\text {eff }}\right),
$$

with the potential

$$
V_{\mathrm{eff}}=\kappa-\frac{2 Q^{2}}{(d-3)(d-2)} e^{-2(d-3)(U+\psi)}-\frac{2 \Lambda}{(d-3)(d-2)} e^{2(U+\psi)},
$$

\footnotetext{
${ }^{3}$ For $d>5$ this does not necessarily imply that the horizon has constant curvature.
} 
if we impose in addition the zero energy condition

$$
e^{2(d-3) \psi}\left(U^{\prime 2}-\psi^{\prime 2}\right)+V_{\text {eff }}=0 .
$$

To be concrete, the equation of motion for $U$ is proportional to the $t t$-component of (2.3), while the one for $\psi$ is a linear combination of the $t t$ - and $r r$-components. Moreover, from the first of (2.3) and the $t t$-component one gets (2.10). The Einstein equations along the fiber are automatically satisfied.

The conjugate momenta and Hamiltonian of the dynamical system (2.8) are respectively given by

$$
\begin{aligned}
p_{U} & =\frac{\partial L}{\partial U^{\prime}}=2 e^{2(d-3) \psi} U^{\prime}, \quad p_{\psi}=\frac{\partial L}{\partial \psi^{\prime}}=-2 e^{2(d-3) \psi} \psi^{\prime}, \\
H_{\mathrm{eff}}\left(p_{U}, p_{\psi}, U, \psi\right) & =\frac{1}{4} e^{-2(d-3) \psi}\left(p_{U}^{2}-p_{\psi}^{2}\right)+V_{\mathrm{eff}} .
\end{aligned}
$$

\section{Integration of the Hamilton-Jacobi equation}

The Hamilton-Jacobi equation associated to (2.11) reads

$$
H_{\mathrm{eff}}\left(\partial_{U} S, \partial_{\psi} S, U, \psi\right)+\frac{\partial S}{\partial r}=0
$$

Since $H_{\text {eff }}$ does not depend explicitely on $r$ we set

$$
S=2 W(U, \psi)-E r
$$

such that (3.1) reduces to

$$
e^{-2(d-3) \psi}\left(W_{U}^{2}-W_{\psi}^{2}\right)+V_{\text {eff }}=E,
$$

where $W_{U}$ and $W_{\psi}$ are respectively the partial derivatives of $W$ w.r.t. $U$ and $\psi$. Inspired by $[28,29]$, we define a new set of coordinates

$$
X=e^{(d-3)(U+\psi)}, \quad Y=e^{-2(d-3) U},
$$

for which (3.3) becomes

$$
\frac{4(d-3)^{2}}{X^{2}}\left(Y W_{Y}^{2}-X W_{X} W_{Y}\right)-\frac{2 Q^{2}}{(d-2)(d-3) X^{2}}-\frac{2 \Lambda X^{\frac{2}{d-3}}}{(d-2)(d-3)}=\hat{E},
$$

where $\hat{E}=E-\kappa$. To avoid loss of information $E$ will be set to zero, as required by (2.10), only at the end of the integration procedure. The reason for this is that, in order to solve the dynamics algebraically, one needs (3.9) and (3.19), therefore we set $E=0$ only after these equations have been obtained. 


\subsection{First solution}

Applying the method of characteristics yields

$$
\frac{\mathrm{d} W_{Y}}{W_{Y}}=\frac{\mathrm{d} X}{X},
$$

and thus $W_{Y}=a X$, where $a$ is an integration constant. The solution of this equation boils down to $W(X, Y)=a Y X+\omega(X)$ that inserted into (3.5) leads to an ODE

$$
-4 a(d-3)^{2} \omega_{X}-\frac{2 Q^{2}}{(d-2)(d-3) X^{2}}-\frac{2 \Lambda X^{\frac{2}{(d-3)}}}{(d-2)(d-3)}=\hat{E},
$$

that can be easily integrated to give

$$
S_{1}=2 a Y X+\frac{1}{2 a(d-3)^{2}}\left(\frac{2 Q^{2}}{(d-2)(d-3) X}-\frac{2 \Lambda X^{\frac{d-1}{d-3}}}{(d-1)(d-2)}-\hat{E} X\right)-E r+C .
$$

This contains three integration constants $C, E$ and $a$, where the latter must be different from zero. Using

$$
\left.\frac{\partial S_{1}}{\partial E}\right|_{E=0}=c_{1},\left.\quad \frac{\partial S_{1}}{\partial a}\right|_{E=0}=c_{2},
$$

where $c_{1}$ and $c_{2}$ denote arbitrary constants, the dynamics can be solved algebraically, with the result

$$
\begin{aligned}
& X=-2 a(d-3)^{2}\left(r+c_{1}\right) \\
& Y=\frac{c_{2}}{2 X}+\frac{Q^{2}}{2 a^{2}(d-2)(d-3)^{3} X^{2}}+\frac{\kappa}{4 a^{2}(d-3)^{2}}-\frac{\Lambda X^{\frac{2}{d-3}}}{2 a^{2}(d-1)(d-2)(d-3)^{2}} .
\end{aligned}
$$

In terms of $Y$ and the new radial coordinate $R=X^{\frac{1}{d-3}}$, the solution (2.4) becomes

$$
\begin{aligned}
\mathrm{d} s_{d}^{2} & =-Y \mathrm{~d} t^{2}+\frac{\mathrm{d} R^{2}}{Y}+R^{2} \mathrm{~d} \Omega_{\kappa, d-2}^{2}, \quad F=\frac{Q}{R^{d-2}} \mathrm{~d} t \wedge \mathrm{d} r \\
Y & =\kappa-\frac{2 M}{R}+\frac{2 Q^{2}}{(d-2)(d-3) R^{2(d-3)}}-\frac{2 \Lambda R^{2}}{(d-1)(d-2)} .
\end{aligned}
$$

Here we fixed $a^{2}=\frac{1}{4(d-3)^{2}}$ (which can always be achieved by rescaling the coordinates appropriately) and defined $c_{2}=-4 M$. (3.11) is the most general solution to the equations of motion following from (2.8), and represents a generalization of the $d$-dimensional Reissner-Nordström-(A)dS black hole to the case where the horizon is an arbitrary Einstein space.

In the original coordinates, Hamilton's characteristic function reads

$$
W_{1}(U, \psi)=a e^{(d-3)(\psi-U)}+\frac{Q^{2} e^{-(d-3)(U+\psi)}}{2 a(d-2)(d-3)^{3}}-\frac{\Lambda e^{(d-1)(U+\psi)}}{2 a(d-1)(d-2)(d-3)}+\frac{\kappa e^{(d-3)(U+\psi)}}{4 a(d-3)^{2}} .
$$

The expressions for the conjugate momenta

$$
p_{U}=2 \frac{\partial W_{1}}{\partial U}, \quad p_{\psi}=2 \frac{\partial W_{1}}{\partial \psi},
$$


together with (2.11), lead to the first order flow equations

$$
U^{\prime}=e^{-2(d-3) \psi} \partial_{U} W_{1}(U, \psi), \quad \psi^{\prime}=-e^{-2(d-3) \psi} \partial_{\psi} W_{1}(U, \psi),
$$

that are satisfied by the nonextremal black holes (3.11). Notice also that, using (3.13), the action (2.8) can be written as a sum of squares. This clarifies also the reason for the very existence of first order equations for nonextremal black holes, namely they are just the expressions for the conjugate momenta in terms of derivatives of the principal function in a Hamilton-Jacobi formalism.

In the BPS case for $d=4$, one would expect to recover the supergravity BPS flow [23], in absence of vector multiplets, that is driven by ${ }^{4}$

$$
W_{\mathrm{BPS}}(U, \psi)=e^{-U} Q+e^{2 \psi+U} g,
$$

where $g$ is related to the cosmological constant by $\Lambda=-3 g^{2}$. However, it is easy to see that there is no limit in which (3.14) can arise from $W_{1}$. We shall come back to this issue in the next subsection.

\subsection{Second solution}

Similar to what was done in [24] for $N=2, d=4$ ungauged supergravity, and in [17] for the abelian gauged case, we introduce the quantity

$$
\mathbb{Q} \equiv e^{2(d-3) \psi} \frac{U^{\prime}+\psi^{\prime}}{d-3}+W
$$

Using (3.13) and the equations of motion following from the action (2.8), one easily shows that $\mathbb{Q}^{\prime}=0$, and thus $\mathbb{Q}$ is a constant of motion that can be used to simplify (3.5). In phase space we have

$$
\mathbb{Q}=\frac{W_{U}-W_{\psi}}{d-3}+W=-2 Y W_{Y}+W
$$

that implies $W(X, Y)=\mathbb{Q}+\sqrt{Y \omega(X)}$. Plugging this into (3.5) one gets the ODE

$$
-4(d-3)^{2} \frac{\partial}{\partial X}\left(\frac{\omega}{X}\right)-\frac{2 Q^{2}}{(d-2)(d-3) X^{2}}-\frac{2 \Lambda X^{\frac{2}{d-3}}}{(d-2)(d-3)}=\hat{E} .
$$

A final integration leads to the solution of the original differential equation $(3.5)^{5}$

$$
S_{2}=2 \mathbb{Q}-E r+2 \sqrt{-4 A X Y+\frac{2 Q^{2} Y}{(d-2)(d-3)^{3}}-\frac{\hat{E} X^{2} Y}{(d-3)^{2}}-\frac{2 \Lambda X^{\frac{2 d-4}{d-3}} Y}{(d-1)(d-2)(d-3)^{2}}},
$$

which has three arbitrary integration constants $\mathbb{Q}, E, A$, but in this case the parameter domain is the whole $\mathbb{R}^{3}$. Using

$$
\left.\frac{\partial S_{2}}{\partial E}\right|_{E=0}=c_{3},\left.\quad \frac{\partial S_{2}}{\partial A}\right|_{E=0}=c_{4},
$$

\footnotetext{
${ }^{4}$ To derive (3.14) from the results of [23], take the prepotential $F=-i\left(X^{0}\right)^{2}$ and a purely magnetic gauging with FI-parameter proportional to $g$.

${ }^{5}$ This solution was already found in [1] and for $\kappa=0$ but with magnetic fluxes switched on in [16].
} 
gives back (3.10), where

$$
a=-\frac{2}{c_{4}}, \quad c_{1}=c_{3}, \quad c_{2}=-\frac{A c_{4}^{2}}{2} .
$$

To complete the comparison we evaluate

$$
\left.\mathbb{Q}\right|_{W_{1}}=-a X Y+\frac{\kappa X}{4 a(d-3)^{2}}+\frac{Q^{2}}{2 a(d-2)(d-3)^{2} X}-\frac{\Lambda X^{\frac{d-1}{d-3}}}{2 a(d-1)(d-2)(d-3)^{2}} .
$$

Plugging the solution (3.10) into the r.h.s. yields $2 \mathbb{Q}=C-a c_{2}$. In terms of $U$ and $\psi, W_{2}$ reads (setting $E=0$ )

$$
W_{2}(U, \psi)=\mathbb{Q}+\sqrt{A e^{(d-3)(\psi-U)}+\frac{2 Q^{2} e^{-2(d-3) U}}{(d-2)(d-3)^{3}}+\frac{\kappa e^{2(d-3) \psi}}{(d-3)^{2}}-\frac{2 \Lambda e^{2(U+(d-2) \psi)}}{(d-1)(d-2)(d-3)^{2}}},
$$

which leads to the first order flow equations

$$
U^{\prime}=e^{-2(d-3) \psi} \partial_{U} W_{2}(U, \psi), \quad \psi^{\prime}=-e^{-2(d-3) \psi} \partial_{\psi} W_{2}(U, \psi) .
$$

(3.22) and (3.13) have different analytic forms, but share the same general class of physical solutions. Notice also that, contrary to $W_{1}$, there is a well-defined limit in which (3.21) reduces to the BPS superpotential (3.14) for $d=4$, by setting $A=0, \Lambda=-3 g^{2}$ and imposing the Dirac-type quantization condition $2 g Q=\kappa$.

The authors of [1] found that the potential (2.9) can be expressed in terms of a superpotential. One easily verifies that their superpotential (2.5) coincides with (3.21) and that eq. (2.4) of [1] is just the Hamilton-Jacobi equation for zero energy. The fact that a nonextremal black hole solution arises from a first order system via a superpotential construction is thus not surprising at all.

\section{Matter-coupled $N=2, d=4$ gauged supergravity}

In this section, we shall discuss possible generalizations of our formalism to $N=2$ supergravity in four dimensions coupled to vector multiplets and with Fayet-Iliopoulos gauging. The analogue of the one-dimensional effective action (2.8) is then given by [17]

$$
S_{\text {eff }}=\int \mathrm{d} r\left(e^{2 \psi}\left(U^{\prime 2}-\psi^{\prime 2}+g_{i \bar{\jmath}} z^{i \prime} \bar{z}^{\prime \prime}\right)-V_{\text {eff }}\right)
$$

with the potential

$$
V_{\mathrm{eff}}=\kappa-e^{-2(U+\psi)} V_{\mathrm{BH}}-e^{2(U+\psi)} V_{g}(z, \bar{z})
$$

where $[17,23]$

$$
V_{\mathrm{BH}}=g^{i \bar{\jmath}} D_{i} Z \bar{D}_{\bar{\jmath}} \bar{Z}+|Z|^{2}=-\frac{1}{2} Q^{T} \mathcal{M} Q, \quad V_{g}=g^{i \bar{\jmath}} D_{i} \mathcal{L} \bar{D}_{\bar{\jmath}} \overline{\mathcal{L}}-3|\mathcal{L}|^{2}
$$

denote respectively the black hole- and scalar potential. In (4.3), $D_{i}$ is the Kähler-covariant derivative, $Z=\langle Q, \mathcal{V}\rangle, \mathcal{L}=\langle\mathcal{G}, \mathcal{V}\rangle$, with the symplectic section $\mathcal{V}$ and the symplectic vectors of charges $Q$ and gauge couplings $\mathcal{G}$. $\mathcal{M}$ is the matrix defined in eq. (2.7) of [17]. Moreover

$$
\langle A, B\rangle \equiv A^{T} \Omega B=A_{\Lambda} B^{\Lambda}-A^{\Lambda} B_{\Lambda} .
$$


Note that the target space of the one-dimensional sigma model (4.1) is equipped with the metric

$$
d \sigma^{2}=e^{2 \psi}\left(-d \psi^{2}+d U^{2}+g_{i \bar{\jmath}} d z^{i} d \bar{z}^{\bar{\jmath}}\right)
$$

and is thus a Lorentzian cone over a special Kähler manifold times a line, as can be seen by setting $\tau=e^{\psi}$. The conjugate momenta and Hamiltonian read

$$
\begin{aligned}
p_{U} & =2 e^{2 \psi} U^{\prime}, \quad p_{\psi}=-2 e^{2 \psi} \psi^{\prime}, \quad p_{i}=e^{2 \psi} g_{i \bar{\jmath}} \bar{\jmath}^{\bar{\jmath}^{\prime}}, \quad \bar{p}_{\bar{\jmath}}=e^{2 \psi} g_{i \bar{\jmath}} z^{i \prime} \\
H_{\mathrm{eff}} & =e^{-2 \psi}\left(\frac{1}{4} p_{U}^{2}-\frac{1}{4} p_{\psi}^{2}+g^{i \bar{\jmath}} p_{i} p_{\bar{\jmath}}\right)+V_{\mathrm{eff}} .
\end{aligned}
$$

If we set $S=2 W-E r$, the reduced Hamilton-Jacobi equation becomes

$$
e^{-2 \psi}\left(W_{U}^{2}-W_{\psi}^{2}+4 g^{i \bar{\jmath}} \frac{\partial W}{\partial z^{i}} \frac{\partial W}{\partial \bar{z}^{\bar{\jmath}}}\right)+V_{\mathrm{eff}}=E .
$$

As was shown for ungauged [24] and gauged supergravity [17], the quantity

$$
\mathbb{Q} \equiv e^{2 \psi}\left(U^{\prime}+\psi^{\prime}\right)+W
$$

is a first integral also in presence of the scalar fields $z^{i} . \mathbb{Q}$ is the Noether charge related to the symmetry

$$
\delta U=U_{\epsilon}-U=\epsilon, \quad \delta \psi=\psi_{\epsilon}-\psi=-\epsilon,
$$

that leaves the potential (4.2) and the action (4.1) invariant (the latter up to boundary terms). In fact, a function $W$, satisfying (4.7) with $E=0$, drives a first order flow

$$
U^{\prime}=e^{-2 \psi} W_{U}, \quad \psi^{\prime}=-e^{-2 \psi} W_{\psi}, \quad z^{i \prime}=2 e^{-2 \psi} g^{i \bar{\jmath}} \frac{\partial W}{\partial z^{\bar{\jmath}}},
$$

and therefore the variation of (4.1) for infinitesimal $\epsilon$ can be written as

$$
\begin{aligned}
\delta S=S\left(U_{\epsilon}, \psi_{\epsilon}\right)-S(U, \psi) & =-2 \epsilon \int \mathrm{d} r\left(e^{2 \psi}\left(U^{\prime 2}-\psi^{\prime 2}+4 g_{i \bar{\jmath}} z^{i \prime} \bar{z}^{\bar{\jmath}^{\prime}}\right)\right) \\
& =-2 \epsilon \int \mathrm{d} r\left(U^{\prime} W_{U}+\psi^{\prime} W_{\psi}+z^{i \prime} \frac{\partial W}{\partial z^{i}}+\bar{z}^{\bar{\jmath}^{\prime}} \frac{\partial W}{\partial \bar{z}^{\bar{\jmath}}}\right) \\
& =-2 \epsilon \int \mathrm{d} r \frac{d W}{d r},
\end{aligned}
$$

which vanishes if we choose appropriate boundary conditions. Note that the transformation (4.9) is generated by the vector field $\partial_{U}-\partial_{\psi}=\partial_{U}-\tau \partial_{\tau}$, which is a conformal Killing vector of the Lorentzian cone (4.5). The fact that $\mathbb{Q}$ is the Noether charge related to (4.9) follows also from the inverse Noether theorem: ${ }^{6}$ if $\mathbb{Q}$ is a conserved charge, then the transformation

$$
\delta q^{I}=\left[q^{I}, \epsilon \mathbb{Q}\right]=\epsilon \frac{\partial \mathbb{Q}}{\partial p_{I}}, \quad \delta p_{I}=\left[p_{I}, \epsilon \mathbb{Q}\right]=-\epsilon \frac{\partial \mathbb{Q}}{\partial q^{I}},
$$

where $[$,$] denotes the Poisson bracket, is a symmetry of the action.$

\footnotetext{
${ }^{6}$ See [31] for a nice review.
} 
As before, we introduce the coordinates

$$
X=e^{U+\psi}, \quad Y=e^{-2 U} .
$$

Then the first integral (4.8) becomes

$$
\mathbb{Q}=-2 Y W_{Y}+W,
$$

which can be easily integrated to give

$$
W(X, Y, z, \bar{z})=\mathbb{Q}+\sqrt{Y \omega(X, z, \bar{z})},
$$

where $\omega$ is an integration 'constant'. Using (4.15), the Hamilton-Jacobi equation (4.7) boils down to

$$
-\partial_{X} \frac{\omega}{X}+\frac{1}{\omega X^{2}} g^{i \bar{\jmath}} \frac{\partial \omega}{\partial z^{i}} \frac{\partial \omega}{\partial \bar{z}^{\bar{\jmath}}}-X^{2} V_{g}-\frac{1}{X^{2}} V_{\mathrm{BH}}+\kappa=E .
$$

A particular solution to (4.16) is the one found in [23] by squaring the action for the BPS case,

$$
\omega_{\mathrm{BPS}}=\left(Z-i X^{2} \mathcal{L}\right)\left(\bar{Z}+i X^{2} \overline{\mathcal{L}}\right)=|Z|^{2}+X^{4}|\mathcal{L}|^{2}-i X^{2}(\mathcal{L} \bar{Z}-\overline{\mathcal{L}} Z)
$$

Imposing $E=0$, as required by Einstein's equations, and using

$$
\frac{\partial \omega}{\partial z^{i}}=\left(\bar{Z}+i X^{2} \overline{\mathcal{L}}\right)\left(D_{i} Z-i X^{2} D_{i} \mathcal{L}\right)
$$

as well as the special Kähler geometry identity

$$
\frac{1}{2}(\mathcal{M}-i \Omega)=\Omega \overline{\mathcal{V}} \mathcal{V} \Omega+\Omega D_{i} \mathcal{V} g^{i \bar{\jmath}} D_{\bar{\jmath}} \overline{\mathcal{V}} \Omega,
$$

it is only matter of some algebra to shew that (4.17) solves (4.16) if one imposes the Dirac charge quantization condition

$$
\langle\mathcal{G}, \mathcal{Q}\rangle=-\kappa .
$$

In the following subsection we shall consider a particular prepotential, for which the effective action (4.1) has additional symmetries, that allow a further reduction of the HamiltonJacobi equation (4.16).

\subsection{Prepotential $F=-i X^{0} X^{1}$}

This simple model has only one complex scalar field $z$ parametrizing the Poincaré halfplane, with Kähler metric

$$
\mathrm{d} s^{2}=\frac{\mathrm{d} z \mathrm{~d} \bar{z}}{(z+\bar{z})^{2}},
$$

which has the three Killing vectors

$$
v_{1}=i\left(\partial_{z}-\partial_{\bar{z}}\right), \quad v_{2}=z \partial_{z}+\bar{z} \partial_{\bar{z}}, \quad v_{3}=\frac{i}{2}\left(\bar{z}^{2} \partial_{\bar{z}}-z^{2} \partial_{z}\right)
$$


These are all symmetries of the ungauged theory, but in presence of a potential for the scalars only a linear combination of them survives, as was shown in [25] using the symplectic representation.

If we consider a configuration with only magnetic charges and purely electric gaugings, the HJ equation (4.16) becomes for this prepotential

$$
-\partial_{X} \frac{\omega}{X}+\frac{1}{\omega X^{2}} g^{z \bar{z}} \frac{\partial \omega}{\partial z} \frac{\partial \omega}{\partial \bar{z}}+X^{2} \frac{g_{0}^{2}+g_{1}^{2} z \bar{z}+2 g_{0} g_{1}(z+\bar{z})}{z+\bar{z}}-\frac{1}{X^{2}} \frac{p^{1^{2}}+p^{0^{2}} z \bar{z}}{z+\bar{z}}+\kappa=E .
$$

The linear combination

$$
v=\frac{g_{0}^{2}}{2 g_{1}^{2}} v_{1}+v_{3}=\frac{i}{2}\left(\frac{g_{0}^{2}}{g_{1}^{2}}-z^{2}\right) \partial_{z}-\frac{i}{2}\left(\frac{g_{0}^{2}}{g_{1}^{2}}-\bar{z}^{2}\right) \partial_{\bar{z}}
$$

generates a symmetry of (4.1) if one imposes the BPS condition [32] $p^{0} g_{0}=p^{1} g_{1}$. It is straightforward to verify that this implies the existence of a further conserved charge

$$
\mathbb{C}=\frac{i}{2}\left(\frac{g_{0}^{2}}{g_{1}^{2}}-z^{2}\right) \frac{\partial \omega}{\partial z}-\frac{i}{2}\left(\frac{g_{0}^{2}}{g_{1}^{2}}-\bar{z}^{2}\right) \frac{\partial \omega}{\partial \bar{z}} .
$$

By introducing the new variables

$$
z=\frac{g_{0}}{g_{1}} \tanh \left(\frac{g_{0}}{g_{1}}(u+i v)\right), \quad \bar{z}=\frac{g_{0}}{g_{1}} \tanh \left(\frac{g_{0}}{g_{1}}(u-i v)\right),
$$

(4.25) can easily be integrated, with the result $\omega=2 \mathbb{C} v+\alpha(u, X)$. Plugging this into (4.23), the HJ equation assumes the form

$$
-\partial_{X} \frac{\alpha}{X}+\left(\frac{g_{1}}{g_{0}}\right)^{2} \frac{\sinh ^{2}\left(2 g_{0} u / g_{1}\right)}{4 X^{2}} \frac{\alpha_{u}^{2}+4 \mathbb{C}^{2}}{\alpha+2 \mathbb{C} v}-X^{2} V_{g}(u)-\frac{1}{X^{2}} V_{\mathrm{BH}}(u)+\kappa=E,
$$

where

$$
V_{\mathrm{BH}}(u)=\frac{\left(p^{0}\right)^{2} g_{0}}{g_{1} \tanh \left(2 g_{0} u / g_{1}\right)}, \quad V_{g}(u)=-\frac{g_{0} g_{1}}{\tanh \left(2 g_{0} u / g_{1}\right)}-2 g_{0} g_{1}
$$

It is easy to see that (4.27) can be satisfied for all $v$ only if $\mathbb{C}=0,{ }^{7}$ so that we have

$$
-\partial_{X} \frac{\alpha}{X}+\left(\frac{g_{1}}{g_{0}}\right)^{2} \frac{\sinh ^{2}\left(2 g_{0} u / g_{1}\right)}{4 X^{2}} \frac{\alpha_{u}^{2}}{\alpha}-X^{2} V_{g}(u)-\frac{1}{X^{2}} V_{\mathrm{BH}}(u)+\kappa=E .
$$

For the prepotential under consideration, the BPS solution (4.17) reads

$$
\omega_{\mathrm{BPS}}(X, z, \bar{z})=\frac{\left(p^{1}+p^{0} z-X^{2}\left(g_{0}+g_{1} z\right)\right)\left(p^{1}+p^{0} \bar{z}-X^{2}\left(g_{0}+g_{1} \bar{z}\right)\right)}{2(z+\bar{z})} .
$$

Imposing $g_{0} p^{0}=g_{1} p^{1}$ and using the coordinates (4.26), this leads to

$$
\alpha_{\mathrm{BPS}}(X, u)=\frac{p^{1}\left(p^{0}-g_{1} X^{2}\right)^{2} e^{4 p^{1} u / p^{0}}}{p^{0}\left(e^{4 p^{1} u / p^{0}}-1\right)} .
$$

\footnotetext{
${ }^{7}$ This sort of 'axion-free' condition is probably related to the special choice of purely electric gaugings and only magnetic charges, so we don't expect that $\mathbb{C}$ vanishes in a more general setting.
} 
It is interesting to note that the variables $X$ and $u$ separate in (4.31). This suggests to use a product ansatz $\alpha(X, u)=\xi(X) \mu(u)$ in order to get something more general than (4.31). Unfortunately, plugging this into (4.29) gives back precisely (4.31). Another possibility is inspired by the comparison with (3.18) (for $d=4$ ), which contains, in addition to quartic, quadratic and $X$-independent terms that appear also in (4.31), a linear piece in $X$ proportional to the constant $A$ that is essentially a nonextremality parameter (or black hole mass). One may thus try

$$
\alpha(X, u)=\sum_{n=0}^{4} \alpha_{n}(u) X^{n},
$$

where (to be still more general) we added a cubic term as well. However, one can check that, using this ansatz in (4.29) leads to an overdetermined system that admits a solution only for $\alpha_{1}=\alpha_{3}=0$, namely (4.31).

It remains to be seen if there exist additional conserved charges associated to hidden symmetries of the action (4.1), that would allow to completely separate the HamiltonJacobi equation (4.7). Note in this context that the transformation (4.9) acts only on $U$ and $\psi$ but not on the scalars $z^{i}$, whereas (4.24) touches only the $z^{i}$ but not the metric components $U$ and $\psi$. There might thus exist (at least for some specific models) more complicated symmetry transformations involving all the dynamical variables. We hope to come back to a systematic analysis of this issue in a future publication.

\section{Final remarks}

In this paper we considered electrically charged static nonextremal black holes in $d$-dimensional Einstein-Maxwell-(A)dS gravity, whose horizon is a generic Einstein space in $d-2$ dimensions. We have shown that for this system the Hamilton-Jacobi equation is exactly integrable and admits two branches of solutions. One of them exhibits a non-simply connected domain of integration constants and does not reduce to the well-known solution for the $d=4$ BPS case. The principal functions generate two first order flows that are analytically different, but support the same general solution. One of the two sets of flow equations corresponds to those found in [1] and (for $d=4$ and $\Lambda=0$ ) in [2]. We clarified thus also the reason for the very existence of first order equations for nonextremal black holes, namely, they are just the expressions for the conjugate momenta in terms of derivatives of the principal function in a Hamilton-Jacobi formalism.

In the last part of our paper we also analyzed if these integrability properties continue to hold for matter-coupled $N=2, d=4$ gauged supergravity. Unfortunately it turned out that the principal function $W$ for nonextremal black holes is not straightforwardly generalizable to this case. Still, we showed (for the example of a particular model) that there exist several conserved charges that allow a partial separation of variables in the HJ equation. These conserved charges comprise the one originally introduced for ungauged supergravity in [24] and subsequently adapted to the gauged theory in [17], as well as those associated to the symmetries recently discovered in [25]. We pointed out the possible existence of additional hidden symmetries of the one-dimensional effective action (4.1) that 
involve simultaneous transformations of the dynamical variables of both the metric and the scalar sector.

One might ask if there exist covariantly constant spinors related to the first order equations. The authors of [2] have shown that the nonextremal Reissner-Nordström solution cannot admit (generalized) Killing spinors in $3+1$ dimensions, but it is supersymmetric in a lower-dimensional effective theory. It might be, however, that the nonextremal black holes considered in this paper possess so-called conformal Killing spinors (CKS, cf. e.g. [33] for a review of this topic). Note in this context that both the (nonextremal) Kerr metric and all other type II-II vacuum spacetimes do admit a CKS [34]. We hope to come back to this point in a future publication.

\section{Acknowledgments}

This work was supported partly by INFN.

Open Access. This article is distributed under the terms of the Creative Commons Attribution License (CC-BY 4.0), which permits any use, distribution and reproduction in any medium, provided the original author(s) and source are credited.

\section{References}

[1] H. Lü, C.N. Pope and J.F. Vazquez-Poritz, From AdS black holes to supersymmetric flux branes, Nucl. Phys. B 709 (2005) 47 [hep-th/0307001] [InSPIRE].

[2] C.M. Miller, K. Schalm and E.J. Weinberg, Nonextremal black holes are BPS, Phys. Rev. D 76 (2007) 044001 [hep-th/0612308] [INSPIRE].

[3] A. Ceresole and G. Dall'Agata, Flow equations for non-BPS extremal black holes, JHEP 03 (2007) 110 [hep-th/0702088] [INSPIRE].

[4] L. Andrianopoli, R. D'Auria, E. Orazi and M. Trigiante, First order description of black holes in moduli space, JHEP 11 (2007) 032 [arXiv:0706.0712] [INSPIRE].

[5] G. Lopes Cardoso, A. Ceresole, G. Dall'Agata, J.M. Oberreuter and J. Perz, First-order flow equations for extremal black holes in very special geometry, JHEP 10 (2007) 063 [arXiv: 0706.3373] [INSPIRE].

[6] B. Janssen, P. Smyth, T. Van Riet and B. Vercnocke, A first-order formalism for timelike and spacelike brane solutions, JHEP 04 (2008) 007 [arXiv:0712.2808] [INSPIRE].

[7] G.L. Cardoso and V. Grass, On five-dimensional non-extremal charged black holes and FRW cosmology, Nucl. Phys. B 803 (2008) 209 [arXiv: 0803.2819] [INSPIRE].

[8] J. Perz, P. Smyth, T. Van Riet and B. Vercnocke, First-order flow equations for extremal and non-extremal black holes, JHEP 03 (2009) 150 [arXiv:0810.1528] [INSPIRE].

[9] A. Ceresole, G. Dall'Agata, S. Ferrara and A. Yeranyan, First order flows for $N=2$ extremal black holes and duality invariants, Nucl. Phys. B 824 (2010) 239 [arXiv:0908.1110] [INSPIRE].

[10] P. Galli, T. Ortín, J. Perz and C.S. Shahbazi, Non-extremal black holes of $N=2, d=4$ supergravity, JHEP 07 (2011) 041 [arXiv:1105.3311] [INSPIRE]. 
[11] S. Barisch, G. Lopes Cardoso, M. Haack, S. Nampuri and N.A. Obers, Nernst branes in gauged supergravity, JHEP 11 (2011) 090 [arXiv: 1108.0296] [INSPIRE].

[12] D. Klemm and O. Vaughan, Nonextremal black holes in gauged supergravity and the real formulation of special geometry II, Class. Quant. Grav. 30 (2013) 065003 [arXiv:1211.1618] [INSPIRE].

[13] A. Gnecchi and C. Toldo, On the non-BPS first order flow in $\mathcal{N}=2 \mathrm{U}(1)$-gauged supergravity, JHEP 03 (2013) 088 [arXiv:1211.1966] [INSPIRE].

[14] A. Gnecchi and C. Toldo, First order flow for non-extremal AdS black holes and mass from holographic renormalization, JHEP 10 (2014) 075 [arXiv:1406.0666] [INSPIRE].

[15] G.L. Cardoso, M. Haack and S. Nampuri, Nernst branes with Lifshitz asymptotics in $\mathcal{N}=2$ gauged supergravity, JHEP 06 (2016) 144 [arXiv:1511.07676] [INSPIRE].

[16] J. Lindgren, I. Papadimitriou, A. Taliotis and J. Vanhoof, Holographic Hall conductivities from dyonic backgrounds, JHEP 07 (2015) 094 [arXiv: 1505. 04131] [INSPIRE].

[17] D. Klemm, N. Petri and M. Rabbiosi, Symplectically invariant flow equations for $N=2$, $D=4$ gauged supergravity with hypermultiplets, JHEP 04 (2016) 008 [arXiv:1602.01334] [INSPIRE].

[18] U. Gürsoy, E. Kiritsis, L. Mazzanti and F. Nitti, Holography and thermodynamics of 5D dilaton-gravity, JHEP 05 (2009) 033 [arXiv:0812.0792] [INSPIRE].

[19] E. Kiritsis and V. Niarchos, The holographic quantum effective potential at finite temperature and density, JHEP 08 (2012) 164 [arXiv:1205.6205] [INSPIRE].

[20] E. Kiritsis, F. Nitti and L.S. Pimenta, Exotic RG flows from holography, Fortschr. Phys. 65 (2017) 1600120 [arXiv:1611.05493] [InSPIRE].

[21] L. Andrianopoli, R. D'Auria, E. Orazi and M. Trigiante, First order description of D=4 static black holes and the Hamilton-Jacobi equation, Nucl. Phys. B 833 (2010) 1 [arXiv: 0905.3938] [INSPIRE].

[22] J. de Boer, E.P. Verlinde and H.L. Verlinde, On the holographic renormalization group, JHEP 08 (2000) 003 [hep-th/9912012] [INSPIRE].

[23] G. Dall'Agata and A. Gnecchi, Flow equations and attractors for black holes in $\mathcal{N}=2 \mathrm{U}(1)$ gauged supergravity, JHEP 03 (2011) 037 [arXiv: 1012.3756] [INSPIRE].

[24] M. Trigiante, T. Van Riet and B. Vercnocke, Fake supersymmetry versus Hamilton-Jacobi, JHEP 05 (2012) 078 [arXiv: 1203.3194] [INSPIRE].

[25] S.L. Cacciatori, D. Klemm and M. Rabbiosi, Duality invariance in Fayet-Iliopoulos gauged supergravity, JHEP 09 (2016) 088 [arXiv: 1606.05160] [INSPIRE].

[26] C. Charmousis, D. Langlois, D.A. Steer and R. Zegers, Rotating spacetimes with a cosmological constant, JHEP 02 (2007) 064 [gr-qc/0610091] [INSPIRE].

[27] M. Astorino, Charging axisymmetric space-times with cosmological constant, JHEP 06 (2012) 086 [arXiv:1205.6998] [INSPIRE].

[28] R.G. Leigh, A.C. Petkou, P.M. Petropoulos and P.K. Tripathy, The Geroch group in Einstein spaces, Class. Quant. Grav. 31 (2014) 225006 [arXiv:1403.6511] [INSPIRE].

[29] D. Klemm, M. Nozawa and M. Rabbiosi, On the integrability of Einstein-Maxwell-(A)dS gravity in the presence of Killing vectors, Class. Quant. Grav. 32 (2015) 205008 [arXiv: 1506.09017] [INSPIRE]. 
[30] B. O'Neill, Semi-Riemannian geometry (with applications to relativity), Academic Press, San Diego U.S.A. (1983).

[31] M. Bañados and I.A. Reyes, A short review on Noether's theorems, gauge symmetries and boundary terms, Int. J. Mod. Phys. D 25 (2016) 1630021 [arXiv:1601.03616] [InSPIRE].

[32] S.L. Cacciatori and D. Klemm, Supersymmetric AdS $S_{4}$ black holes and attractors, JHEP 01 (2010) 085 [arXiv:0911.4926] [INSPIRE].

[33] H. Baum, Conformal Killing spinors and special geometric structures in Lorentzian geometry: a survey, math/0202008 [INSPIRE].

[34] M. Walker and R. Penrose, On quadratic first integrals of the geodesic equations for type $\{22\}$ spacetimes, Commun. Math. Phys. 18 (1970) 265 [InSPIRE]. 